\title{
Relevance factors of mathematical education for sustainable development
}

\author{
Yury Melnikov*, and Yelena Ivukina \\ Ural State University of Economics, 620144 Ekaterinburg, Russia
}

\begin{abstract}
Education system is viewed as an important constituent of sustainable development of a society. The spread of information technology reduces the need for mastering the computing apparatus of mathematics. On the other hand, firstly, the role of mathematics is not limited at least to its computing apparatus, the other components of the mathematical apparatus (for example, conceptual, methodological, etc.) and other aspects of mathematics (historical, activity, education of "intellectual honesty", the formation of specific types of thinking, for example, geometric, ampect, linked with modelling, etc.) are also significant issues; secondly, the use of a computing apparatus is actually the final stage of other types of mathematical and "near-mathematical" activities. The article identifies three factors that ensure the relevance of mathematical education for the sustainable development: 1) the system of teaching mathematics should form the ability to use the mathematical apparatus for solving problems of business, management, engineering, social development, etc. (five components of this factor are highlighted); 2) focus on working with educational material at the level of typical strategies and methodology, not limited to typical algorithms; 3) presentation of mathematics as a multidimensional cultural phenomenon, in which scientific, technical, aesthetic, historical and economic as well as historical and social aspects are distinguished (this in many cases requires the processing of the teaching methods constituents, in particular, curricula alongside with the educational and methodological support).
\end{abstract}

\section{Introduction}

The role of the education system in ensuring the stable development of a society cannot be overemphasized. The education system, like the health system, is subject to conflicting demands: on the one hand, they must be the conservative institutions themselves, since errors in their framework are extremely dangerous and the consequences are delayed, on the other hand, because of this they lag the requirements of time and constantly need reform. Reforming should contribute to ensuring the sustainable development of a society: science, culture, education, etc. which are necessary for the sustainable society. In this article we pointed out the factors of mathematical education, necessary for the sustainable development of a society.

\footnotetext{
* Corresponding author: UriiMelnikov58@gmail.com
} 


\section{Research methodology}

The research methodology includes the author's theory of modelling by Y. B. Melnikov, based on a formal constructive interpretation of the model, implementing a systematic approach to modelling and including the "algebra of models", the theory of adequacy, the theory of strategies and a system of basic models.

\section{Research results}

The modern world is characterized by an unprecedented speed of technology development, resulted in a high rate of requirement changes not only on the labour market, but also a set of qualities necessary for performing everyday functions (for working with the public services portal, for making safe and economical purchases on the Internet, for organizing effective communication using messengers and social networks, etc.). Therefore, the education system must provide a high adaptability of graduates to changing conditions and requirements. This leads to the following requirements for getting teaching mathematics results.

The system of teaching mathematics should form the ability to apply the mathematical apparatus to problem - solving namely in business, management, engineering, social development, etc. For this it is necessary:

- to form a culture of work with the conceptual apparatus, in particular, to improve the ability of formulating information by means of the corresponding conceptual apparatus (financial problems should be formulated by terms of financial science, problems with staffing - by HR-management terms, etc.);

- to develop the ability of building models of various aspects of the prototype, see Fig. 1, to present information in the form of a model system, primarily mathematical and computer ones;

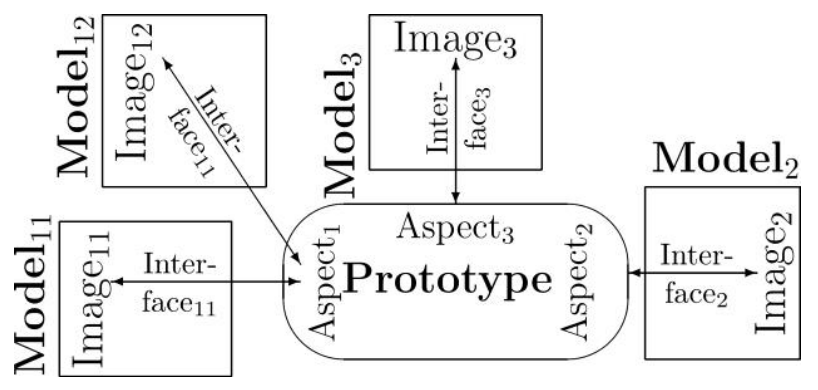

Fig. 1. Multidimensional modelling.

- to improve the ability of analyzing mathematical models, in particular, with the help of information technologies, see Fig. 2.

- to form the ability of interpreting the results of the mathematical model analysis in the original subject area terms;

- to form the ability to carry out a multifaceted assessment of the model adequacy and the results of their analysis, see Fig. 3. 


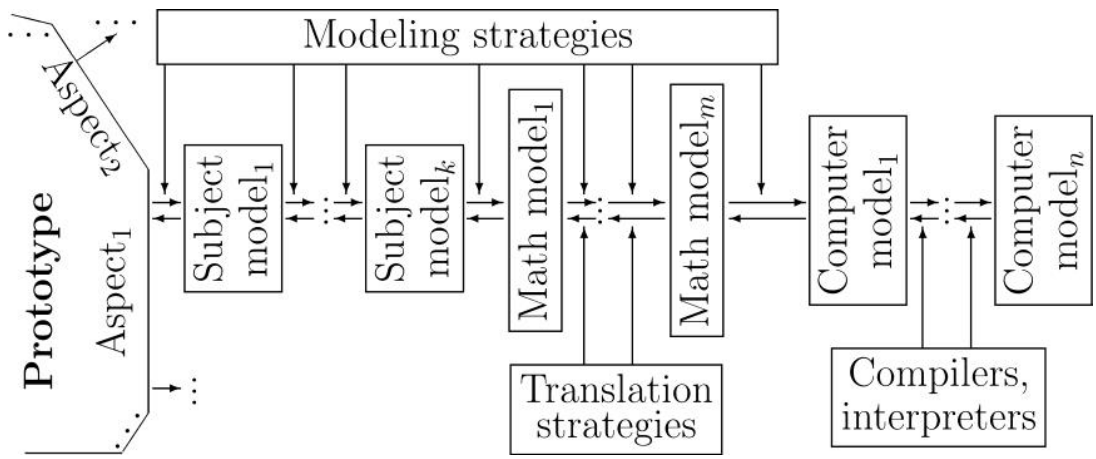

Fig. 2. Simulation as a series of model transformations.
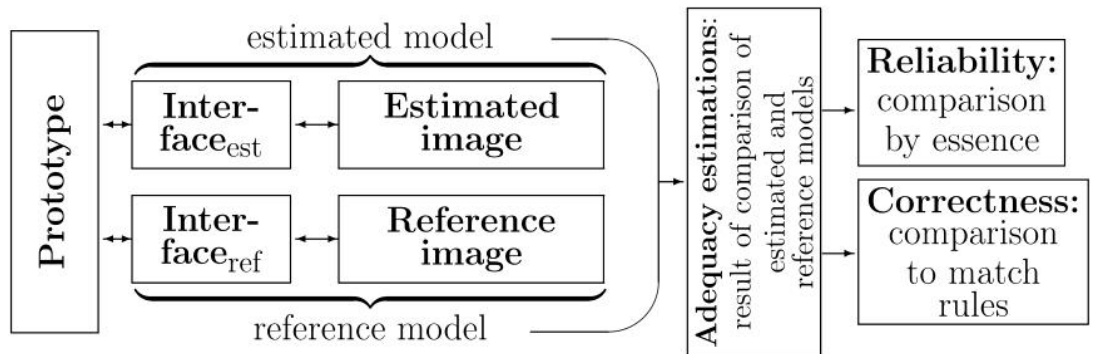

Fig. 3. Adequacy assessment as a result of the evaluated and reference models' comparison

Ensuring a high adaptability to the graduates' changes of educational institutions contradicts to their orientation algorithms implementation, which is often observed in teaching mathematics. We distinguish three levels of dealing with information: II.1) the level of typical algorithms implementation; II.2) the activity strategies level, specific and typical for the given activity area [1]; II.3) the methodology level, characterized by the use of universal strategies (beyond the scope of the studied academic discipline) and the creation of new strategies, primarily by combining strategies and adapting known strategies to new tasks $[2,3]$. We consider strategy as a mechanism for creating the activity plans, see Fig. 4.

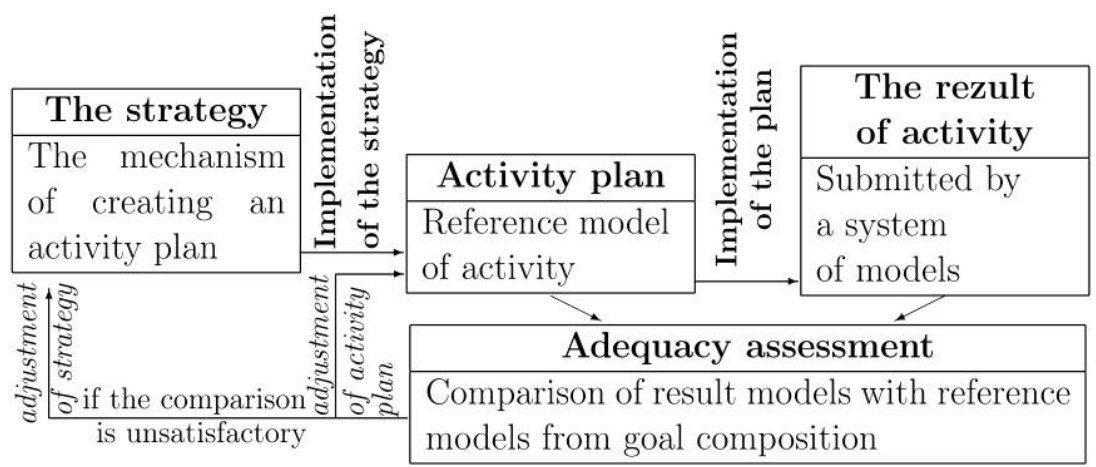

Fig. 4. The relationship among strategy, strategy implementation, activity plan and plan accomplishment.

A characteristic feature of working at level II.1 (the level of algorithms) is, for example, the statement "we have not solved such problems yet". The activity of the subject with formed ability to work at level II.2 (the level of typical strategies), is distinguished by significantly greater flexibility, orientation towards a thorough analysis of the activity goal and available resources. For example, instead of memorizing a large number of formulas, it is worth 
assimilating their derivation. In particular, an alternative to mechanical mugging up of the homogeneous formulas for converting products like $\cos x \cdot \sin y, \sin x \cdot \sin y$, etc. into the sum of sines and cosines is the reasoning carried out in the mind: a) the expression $\cos x \cdot \sin y$ is an integral part of the decomposition formula of the sine sum and angular difference of $x$ и $y$; b) the decomposition formula will be written in short form:

$$
\sin (x+y)=\sin x \cdot \cos y+\cos x \cdot \sin y=c c+s s
$$

i.e.

$$
\left\{\begin{array}{l}
\sin (x+y)=s c+c s \\
\sin (x-y)=s c-c s
\end{array} \Rightarrow 2 c s=\sin (x+y)-\sin (x-y)\right.
$$

leading to

$$
\cos x \cdot \sin x=\frac{1}{2}(\sin (x+y)-\sin (x-y)) .
$$

Thus, formulas memorization (action "according to the algorithm") is replaced by the abbreviated (oral!) implementation strategy in terms of the formula derivation. It is worth pointing out several features of working at the level of strategies (a reference to II.2) from the algorithm's implementation (a reference to II.1). Firstly, as an axiom, we accepted the statement that each point of the activity plan can be perceived by the developer of the plan and its executor either as a reference to an available algorithm of activity, or as a reference to a (secondary) activity goal without specifying the method of its achievement. The implementation of strategies differs from the algorithm one by using plans, in which the executor perceived some of the plan' points as a reference to a (secondary) goal; consequently, the strategy implementation does not guarantee the goal achievement.

Secondly, unlike the algorithm accomplishment, the strategy implementation is often accompanied by errors, moreover, some strategies are based on the deliberate performance of actions that could be interpreted as errors: preliminary consideration of special cases (application of unreasonable assumptions), reasoning "by contradiction", in particular, obtaining consequences from unproven hypotheses, etc. Thus, teaching the strategies implementation, in contrast to teaching the algorithms performing, requires a balanced attitude to the mistakes of the trainees, they should not be perceived unambiguously as negative ones.

Thirdly, when working at the level of strategies implementation, activity plans, as a rule, are viewed as more compact and flexible, since a) the description of the activity goal is usually more compact in comparison with the algorithm description for achieving it, b) the performer of the plan, completing the point of the plan that he perceived as a reference to the activity goal, can choose a method of achieving it that most closely match the resources available at that moment; c) to implement the strategy, the performer should possess higher qualifications than to accomplish the ready algorithm, so in this case the plan can be free from unnecessary explanations.

Finally, the activity at level II.3 (the methodology level) requires the formation of special cognitive structures responsible for adapting strategies to new tasks, combining strategies, and developing new strategies. Even a brief overview of the relevant methods is far beyond the scope of this article. As an example of working at the methodological level, we consider a strategy for solving equations, the study of which can replace memorizing a large number of equation types and solution methods: 
- start and finish the solution of the equation and inequality by analyzing the tolerance region (TR) of variables and unknowns;

- the goal is to convert to an equation with a known solution algorithm, for example, to a quadratic equation or to the form $p(q(x))=b$, where $p$ is some elementary function, i.e. power, exponential, logarithmic, trigonometric, arc- trigonometric one;

- To achieve the main goal, firstly, identical transformations of algebraic expressions are used, i.e. transformations that do not change their values; secondly, equivalent transformations of equalities; thirdly, the transition to the equation-consequence; fourthly, reducing the equation to the form "product is equal to zero"; fifthly, converting to the form $p(x)=C$, where the value of $C$ is "reached in a jump", i.e. is viewed as extreme for $p$; sixthly, partition into special cases (for example, when solving equations with a modulus); seventhly, changing of variables.

The implementation of paragraphs II.3b and II.3c often requires the known strategies adaptation, the strategies combination, or even the special strategy creation. In the research papers and teaching practice, the transition to strategies and methodology in the study of geometry is considered [4]. To work at the methodological level, we include training of metacognitive strategies [5], self-regulated learning strategies [6], training in analytical and synthetic activities [7], development orientation to the level of epistemology [8], introspection of working with signs [9], challenge-based learning [10], partly - inquiry-based learning [11]. Some problems of the formation and implementation of strategies are discussed in [12], teaching and studying the nature of scientific knowledge and scientific research [13] is also associated with work at the level of strategies and methodology. The use of information technology for teaching work at the level of strategies and methodology is partly discussed in $[14,15]$. Training at the strategies and methodology levels is required to deal with the robotic - cognitive adaptive system R-Castle [16].

In teaching it is necessary to consider mathematics as a multifaceted, multidimensional, cultural phenomenon, possessing scientific, technical, aesthetic, historical and economic as well as historical and social aspects.

At present, we implement this idea by formalizing mathematics models through their different facets: mathematics as a field of activity (see Fig. 5), as a system of mathematical phenomena, as a system of processes, a hardware model of mathematics, historical models of mathematics (phenomenological, personification, paradigmatic), etc.

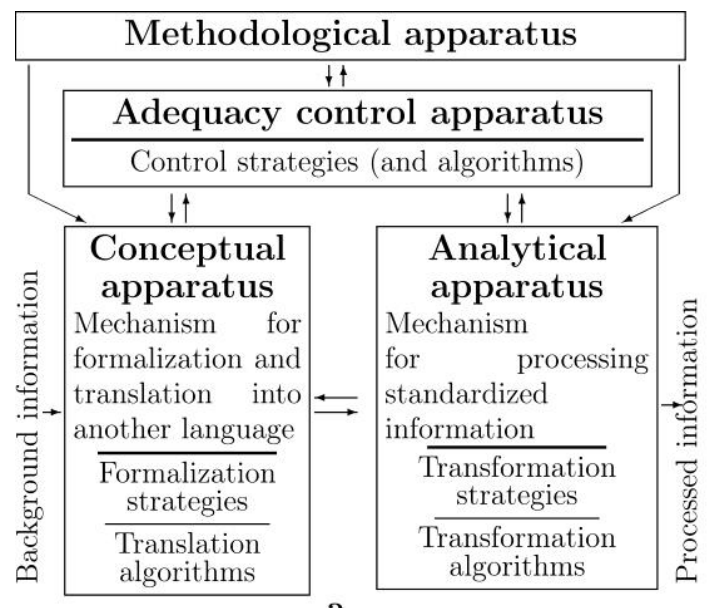

a

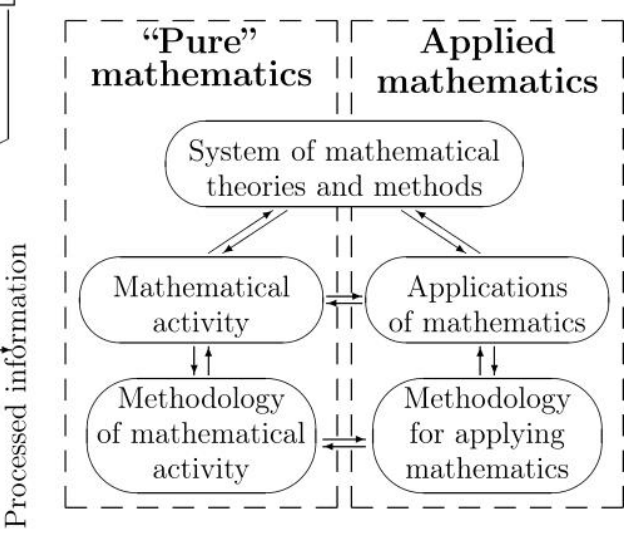

b

Fig. 5. Models of mathematics a) hardware model of mathematics; b) mathematics as a field of activity. 
Various aspects of mathematics are revealed when teaching so-called "soft skills" [17], as well as implementing STEM-learning (Science, Technology, Engineering and Mathematics) $[18,19,20,21]$ and other options for combining academic disciplines, for example, COMPASS (Coordinated Math-Physics Assessment for Student Success) [22]. It is noted that teaching mathematics is associated with comprehension-oriented learning of texts [23] (it is worth mentioning mathematical texts, whereas the plot problem does not actually relate to mathematics).

According to our observations, not enough attention is paid to the item I.1in teaching practice) "to form a culture of work with the conceptual apparatus." Firstly, definitions and theorems are often formulated in a form not very suitable for the use in mathematical activity. For example, it is very difficult to use the statement uniqueness in the conclusion of the theorem "under such conditions there is a unique solution to the Cauchy problem in this framework $\mathrm{U}(\mathrm{x} 0)$ ". The fact is that the most powerful apparatus in mathematics is developed for processing equalities, a slightly weaker one - for processing inequalities and set-theoretic inclusions. In order to formulate a uniqueness statement in the "language of equalities", one can apply a strategy of information formalizing. One of its basic sub-strategies is the strategy of anticipation [3], aimed at building a desired result model, an initial state model and a plan of the corresponding transformations. Sometimes this model is used in a "skewed" way, when the desired result is replaced by its opposite one. In other words, it is often easier to formulate a negation to a formalized statement in the required language, and then construct a formal negation. For example, the presence of two different solutions to the Cauchy problem $\left\{\begin{array}{c}y^{\prime}=f(x, y), \\ y\left(x_{0}\right)=y_{0}\end{array}\right.$ can be written as follows: $\left\{\begin{array}{c}p^{\prime}=f(x, p(x)), \\ q^{\prime}=f(x, q(x)), \text { but } \exists x \in U\left(x_{0}\right)(p(x) \neq \\ p\left(x_{0}\right)=q\left(x_{0}\right)=y_{0},\end{array}\right.$ $q(x))$.. Then negation can be represented as: $\left\{\begin{array}{c}p^{\prime}=f(x, p(x)), \\ q^{\prime}=f(x, q(x)), \quad \\ p\left(x_{0}\right)=q\left(x_{0}\right)=y_{0}\end{array} \Rightarrow \forall x \in U\left(x_{0}\right)(p(x)=\right.$ $q(x))$

Another example of the concept formalization is the following: to formulate a mathematical definition of the coordinate system of the region $U$ on the plane. In this case, it is advisable to apply the strategy of building a model [2]. The use of the coordinate system will be viewed as the modeled aspect of the prototype. The plane coordinate system enables any ordered pair of numbers to map to a point on the plane. In mathematics, this situation is modeled by the concept of "function" in a standard way, considered as a one-to-one mapping of one set into another (we do not consider multivalued functions here). So, the generic concept for the coordinate system is the concept of "function". It remains to determine the characteristic properties of the coordinate system precisely. It is worth referring to the basic characteristics of a function: definition domain and values domain. As a result, we get, for example, such a formulation; "The coordinate system of the domain $U$ of the plane $P$ is a function which definition domain is included in $\mathbb{R} \times \mathbb{R}$ and the range of values $U \subseteq P$.." Thus, the concept formalization in the form of a definition is usually based on the implementation of the following activity stages: 1) the allocation of the scope of the defined concept; 2) the choice of a generic concept; 3 ) the formation of key statements from its content; 4) the formulation with their help of a characteristic property that distinguishes objects from the scope of the defined concept in the scope of the generic one.

In addition to knowledge of definitions and the ability to formalize a concept in the form of a definition, an important component of the culture of working with a conceptual apparatus is the ability of using the definition to solve problems (not only in mathematics). 
For example, consider the problem "find a function $s$ for which the identity $s(3-2 t)=$ $4 t-t^{2} "$ holds. It is natural to start solving this problem by defining a function as an unambiguous mapping formulated in the "language of equalities, inequalities, and settheoretic inclusions": $\left\{\begin{array}{c}a \in D(s), \\ b \in D(s), \Rightarrow s(a)=s(b) \text {.. In this case, using the identity from the } \\ a=b\end{array}\right.$ problem statement, we obtain: $\left\{\begin{array}{c}3-2 t \in D(s), \\ x \in D(s), \\ 3-2 t=x\end{array} \Rightarrow s(3-2 t)=s(x)=4 t-t^{2}\right.$. Expressing $t$ in terms of $x$, we get the answer: $s(x)=2(3-x)-\left(\frac{3-x}{2}\right)^{2}$..

Very little attention is also paid to the Clause I.2) "develop the ability to build models of various aspects of the prototype", in the practice of teaching mathematics. For example, the authors do not know any school mathematics textbook that would describe various aspects of the theorem under consideration: as an activity object (the formulation of the theorem must be understood, memorized, the premise and conclusion should be highlighted, and equivalent formulations should be built), as an instrument of activity, as a cultural phenomenon, as culturally and aesthetically significant. phenomena, etc. In the educational literature, the aesthetic aspect of mathematics is usually almost not reflected, phrases such as a "fruitful result", "effective method", "elegant technique", etc. are rarely found. For example, the aesthetic aspect of the Pythagorean theorem may be determined by the result unexpectedness. Usually, the study of the Pythagorean theorem begins with its formulation. In our opinion, this loses the effect of surprise, the attractiveness perception of this theorem. For instance, you can ask the trainees first to formulate independently a hypothesis about the relationship between the squares of the side lengths. The teacher can either, after several options, write the correct conclusion of the theorem on his own, or choose it from the proposed ones, or interpret the squares of numbers as the area of the corresponding squares and, as a result of the construction, analysis and enrichment of the drawing, first actually obtain the proof of the theorem (in the form of equality of the areas of the figures), and then interpret geometric relation as the numerical equality. Then you can either show the naturalness of the study subject or begin the study of the Pythagorean theorem by choosing this subject of study. It is not difficult to justify the choice of the study subject. It is advisable to start the process of discussion with the students from what we know at this point about the triangles. Basic values have been introduced: the sides lengths, the angles magnitudes, and some combinations: the sum of all the angles of the triangle is equal to the value of the unfolded angle, the sum of the lengths of the sides, i.e. perimeter, characterizing the triangle size, another characteristic of the size is the area. It leads to continue combining values. Summing up the lengths and angles is geometrically meaningless (you can get a numerical value, but how can you interpret it geometrically?). All that remains is multiplication. Multiplying the angles is also geometrically meaningless: what do square degrees measure (there is no use of talking about solid angles yet)? So, you can try to multiply the lengths of the sides. The most promising strategy looks like a priority study of extreme situations (it is better not to pronounce the word "strategy" itself, but it is highly desirable to form an idea of it): firstly, it is most fruitful when both factors coincide (that is, to consider the squares of the sides lengths), secondly, one should confine oneself to "extreme triangles": isosceles or rectangular ones (in the latter, the maximum is the angle between the straight lines, which are the legs continuation). The choice in favor of a right-angled triangle can be made by the teacher directly.

The implementation of the clause I.3) "to improve the ability of analyzing mathematical models, in particular, using information technologies" is currently simplified by the emergence of such free software as the Maxima package, Libre Office Calc, Gap, etc. Our 
experience in laboratory work (using computers running Windows or various versions of Linux, as well as smartphones on Android and Maxima for Android programs), we evaluate positively on the whole, despite the identified shortcomings, the majority of which we plan to eliminate in future.

\section{Discussion of the results}

Over the centuries (and even millennia) mathematics has gained a growing authority, primarily due to the rapidly improving computing apparatus. With the spread of information technologies, the computing apparatus is increasingly "migrating" into the software of both professional activity and everyday life, entertainment, etc. This requires a rethinking of the role and place of mathematical education.

\section{Conclusions}

In this paper, we have proposed three directions for adapting mathematics education to the conditions when the computing apparatus of mathematics is increasingly integrated into software.

The system of teaching mathematics should form the ability of applying the mathematical apparatus to problem-solving namely in business, management, engineering, social development, etc. Wherein, teaching modeling is not limited to solving "applied", "text", "plot" problems, it is much more efficient to build models (mathematical and nonmathematical) of mathematical phenomena.

Training should be focused on managing activities through the strategies implementation. We have identified three levels of working with information:

- the level of typical algorithms implementation;

- the activity strategies level, specific and typical for the given activity area];

- the methodology level, characterized by the use of universal strategies (beyond the scope of the studied academic discipline) and the creation of new strategies, primarily by combining strategies and adapting known strategies to new tasks.

The system of teaching mathematics should reflect various aspects of mathematics as a multidimensional cultural phenomenon: scientific, technical, aesthetic and other ones.

\section{References}

1. Y. B. Melnikov, S. M. Privalov, Modern Educ., 4, 1 (2019)

2. J. B. Melnikov, K. S. Potorochina, Yaroslavl Pedagogical Bull., 3, 19 (2010)

3. Y. B. Melnikov, I. B. Khripunov, B. S. Chopovda, J. Ural State Univ. Econ., 2, 115 (2014)

4. S. Llinares, F. Clemente, Math. Educ. Res. J., 31, 259 (2019)

5. E. I. Perikova, A. E. Loviagina, V. M. Byzova, Sci. Educ. Today, 9, 19 (2019)

6. B. Zheng, A. Ward, R. Stanulis, Med. Educ. Online, 25, 1686949 (2020)

7. I. B. Shmigirilova, A. A. Chugunova, N. I. Pustovalova, Sci. Educ. Today, 9, 121 (2019)

8. S. Hussmann, F. Schacht, M. Schindler, Math. Educ. Res. J., 31, 133 (2019)

9. A. M. Wille, Int. J. Sci. Math. Educ., 18, 1587 (2019)

10. L. C. Felix-Herran, A. E. Rendon-Nava, J. M. Nieto Jalil, IJIDEM, 13, 1367-1383 (2019)

11. T. A. Eppes, I. Milanovic, K. Wright, iJOE, 16, 4-17 (2020) 
12. C. Randall, E. B. Dent, J. Manag. Hist., 25, 401-427 (2019)

13. N. G. Lederman, J. S. Lederman, J. Sci. Teach. Educ., 30, 737-762 (2019)

14. S.-Y. Wu, J. Educ. Comp. Res., 58, 249-272 (2020)

15. A. Clark-Wilson, C. Hoyles, Educ. Stud. Math., 102, 343-359 (2019)

16. D. Tozadore, A. Pinto, J.Valentini, M. Camargo, R. Zavarizz, V. Rodrigues, F. Vedrameto, R. Romero, IEEE TCDS, 11, 581-589 (2019)

17. I. K. Tsalikova, S. V. Pakhotina, Education and Science, 21, 187-207 (2019)

18. J. Miller, ZDM, 51, 915-927 (2019)

19. J. Gil-Quintana, V. Malvasi, B. Castillo-Abdul, L. M. Romero-Rodriguez, Sustainability, 12, 7466 (2020)

20. E. S. Park, F. Ngo, T. Melguizo // Res. High. Educ., 62, 403-447 (2020)

21. F. Alegre, L. Moliner, A. Maroto, G. Lorenzo-Valentin, Educ. Rev., 71, 767-791 (2019)

22. G. Yao, K. Black, M. W. Ramsdell, M. K. Voigt, K. K. Rubasinghe Kattadige, W. Li, ASEE Annual Conf., 127, 34334 (2020)

23. E. Kikas, K. Mädamürk, A. Palu, Br. J. Educ. Psychol., 90, 105-123 (2020) 